\title{
A multiscale investigation on the thermal transport in polydimethylsiloxane nanocomposites: graphene vs borophene
}

\author{
Alessandro Di Pierro ${ }^{a}$, Bohayra Mortazavi ${ }^{b *}$, Hamidreza Nooric, Timon Rabczuk ${ }^{c}$ and Alberto Fina ${ }^{a * *}$ \\ aDipartimento di Scienza Applicata e Tecnologia, Politecnico di Torino, Alessandria Campus, Viale Teresa Michel 5, 15121 \\ Alessandria, Italy. \\ ${ }^{b}$ Department of Mathematics and Physics, Leibniz Universität Hannover, Appelstraße 11, 30167 Hannover, Germany. \\ 'Institute of Structural Mechanics, Bauhaus-Universität Weimar, Marienstr. 15, D-99423 Weimar, Germany
}

\begin{abstract}
Graphene and borophene are highly attractive two-dimensional materials with outstanding physical properties. In this study we employed a combined atomistic continuum multiscale modeling to explore the effective thermal conductivity of polymers nanocomposites made of PDMS polymer as the matrix and graphene and borophene as nanofillers. We first conduct classical molecular dynamics simulations to investigate the interfacial thermal conductance between graphene/PDMS and borophene/PDMS interfaces. Acquired results confirm that the interfacial thermal conductance between nanosheets and polymer increases from the single-layer to multilayered nanosheets and finally converges. The data provided by the atomistic simulations were then used in the finite element method simulations to evaluate the effective thermal conductivity of polymer nanocomposites at continuum level. We explore the effects of nanofillers type, their volume content, geometry aspect ratio and thickness on the nanocomposites effective thermal conductivity. As a very interesting finding, we show that borophene nanosheets, despite almost two orders of magnitude lower thermal conductivity than graphene, can yield very close enhancement in the effective thermal conductivity in comparison with graphene, particularly for low volume content and small aspect ratios and thicknesses. We conclude that for the polymer-based nanocomposites, significant improvement in the thermal conductivity can be reached by improving the bonding between the fillers and polymer or in another word enhancing the thermal conductance at the interface. By taking into account the high electrical conductivity of borophene, our results suggest borophene nanosheets as promising nanofillers to simultaneously enhance the polymers thermal and electrical conductivity.
\end{abstract}

\section{Keywords:}

Thermal transport in nanocomposites; interfacial thermal conductance; graphene; borophene; multiscale modelling of thermal transport.

Corresponding authors: *bohayra.mortazavi@gmail.com; ${ }^{* *}$ alberto.fina@polito.it 


\section{Introduction}

Thermal management is currently a critical in several advanced and rapidly growing technologies, like electronics and electrified vehicles. In fact, inappropriate thermal managements can result in sudden or local rise of hot spots, which may not only substantially shorten the device life but can also lead to hazardous conditions. In recent years, the development of flexible devices, such as wearable technologies open new challenges in thermal management, since traditional solutions effectiveness becomes limited. After graphene has been successfully isolated in 2004 [1, 2], two-dimensional (2D) materials started to attract tremendous attention. For the case of graphene, it exhibits outstanding physical properties, including ultrahigh thermal, mechanical and electronic carrier mobility. Uniquely high properties of graphene along with its outstanding flexibility, proposed this nanomaterial as a highly promising candidate for the employment in thermal management systems. Within 2D materials, borophene $[3,4]$ nanosheets have been recently fabricated as a graphene competitor due to their strong bonds and appealing electronic and magnetic properties. Among the various approaches to exploit the exciting properties of 2D materials is to disperse them inside a continuous matrix made of polymer, creating a composite material. Although the thermal conductivity of graphene polymer nanocomposites has been extensively studied in the literature [5], borophene polymer nanocomposites are basically unexplored, either theoretically or experimentally. In order to improve the knowledge with respect to the application of borophene in thermal management systems, in this work we conduct a multiscale theoretical study to compare the graphene and borophene for the employment as the reinforcement nanomaterials for the improvement of the thermal conductivity of polymeric materials.

The polymer chosen for this investigation is Polydimethylsiloxane (PDMS), which has been reported in the literature to strongly interact with graphene nanomembranes [6]. Moreover, based on previous reports, PDMS was found application-relevant for its set of properties such as a low glass transition temperature (thus flexibility), chemical inertia, and a wide range of physical states, from liquid to rubbery state. The improvement of thermal conductivity in PDMS matrix composites thus represent an alluring solution for heat transfer for several applications, including highly deformable devices. Worthy to note that the thermal conductivity of PDMS and graphene composites was experimentally investigated by several authors, with findings strongly correlated to the specific structure. Zhao and coworkers [7] found that a $0.7 \%$ graphene content in weight can enhance the thermal conductivity of PDMS based composite by more than twice, passing from $0.19 \mathrm{~W} \mathrm{~m}^{-1} \mathrm{~K}^{-1}$ of neat PDMS to about $0.45 \mathrm{~W}$ $\mathrm{m}^{-1} \mathrm{~K}^{-1}$ of graphene sheet composite. Such remarkable improvement was attributed to the creation of a tight percolation network of graphene platelets. The platelets employed from Zhao and coworkers [7] were obtained by foaming, a technique that allowed the creation of particles with length in the range of some micrometers by about $3 \mathrm{~nm}$ of thickness. In 2017, Tian [8] and coworkers adopted silicone rubber (SR) with three different graphene platelets concentrations obtained by mechanical blending and curing. The platelets were about $3 \mathrm{~nm}$ thick and about 5 by 10 micrometers in lateral size. The maximum concentration of graphene platelets, $0.72 \%$ in weight determined an increase of the thermal conductivity from $0.2 \mathrm{~W} \mathrm{~m}^{-1} \mathrm{~K}^{-1}$ of SR to $0.3 \mathrm{~W} \mathrm{~m}^{-1} \mathrm{~K}^{-1}$. The comparison between the work of Zhao [7] and Tian [8] pointed out how the particle displacement and consequently interaction between particles impacts on the composite thermal conductivity. Li and coworkers [9] reviewed several works on 
graphene materials, featured by oriented particles, three dimensional structures or segregated particles, evidencing how the thermal conductivity enhancement in three dimensional structures is about five times than segregated structures. Despite this consideration, high thermal conductivity materials made of segregated particles as the thermoplastics realized by Alam and coworkers [10] or the epoxy resin from Shahil and Balandin [11] pays the drawback of filler loadings in the order of magnitude of one tenth or more.

In this work, our objective is to explore the effectiveness of borophene, as a novel and highly important nanomaterial for the enhancement of polymers thermal conductivity. For this goal we conduct a combined atomistic continuum multiscale modeling. This approach includes classical molecular dynamics simulations to estimate the interfacial thermal conductance that rises between polymer and layered borophene. In the next stage, the estimated interfacial thermal conductance was used as a parameter into the finite element method (FEM) simulations to quantify the effective thermal conductivity of polymer nanocomposites. We assess the effects of as nanofillers' content, thickness and aspect ratio effective on the resulting thermal conductivity. In order to provide a more useful vision, we also employ the developed approach for the case of graphene nanosheets. Our results suggest borophene nanosheets as promising candidates for the improvement of polymers thermal conductivity.

\section{Computational methods}

The Large-scale Atomic/Molecular Massively Parallel Simulator (LAMMPS) [12, 13] package was used to perform all the classical molecular dynamics (MD) simulations All the interatomic forces within the PDMS polymer were calculated by the COMPASS force field. As the most accurate choice to study the thermal conductivity of graphene, the optimized Tersoff [14] was employed to define the carboncarbon bonding interactions in graphene. Interactions between boron atoms in borophene are simulated using the ReaxFF [15] reactive force field. The Van der Waals non-bonded interactions were simulated using the Lennard-Jones (LJ) potential. The pair interactions for equilibrium distance and potential well depth were then calculated, respectively, by arithmetic and geometric mean of the native atoms taken from Universal Force Field [16]. We adopted a small time-step of 0.25 fs in our MD calculations. The reach the densely packed PDMS, the system was first equilibrated using Nosé-Hoover thermostat (NVT) method at $300 \mathrm{~K}$ and then was heated up to $1000 \mathrm{~K}$ to allow the rearrangement of atomic position. The system as next cooled to room temperature (300 K) with the Nosé-Hoover barostat and thermostat (NPT) method. After obtaining the homogenous and bulk PDMS, the periodicity was removed along the $Z$ direction and multi-layer graphene or borophene films were placed on the surface of PDMS polymer. The final models are simulated with periodic boundary conditions along all three Cartesian directions. Finally, we used NPT method to reach geometry optimized structures at the room temperature and atmospheric pressure.

Effective thermal conductivities of PDMS based nanocomposites were obtained using the finite element method. We used ABAQUS/Standard software along with python subroutines for the modeling of nanocomposites samples. In these calculations, representative volume elements (RVEs) were simulated. As an acceptable assumption, the nanofillers were modelled as flat disks dispersed randomly inside a PDMS polymer matrix. In this model, the geometry of the filler is taken into account by tuning the aspect ratio of the disks $(25,50,75,100)$, defined as the diameter to thickness ratio of the plate. 
Within this RVE, no particle-particle contact was allowed and neither disk bending. The heat transfer elements (DC3D4) with 4-node linear tetrahedron shape were used in our calculations.

\section{Results and discussions}

It should be first noted that there are several reports that in layered 2D materials, the interfacial thermal conductance between nanosheets and substrate can be affected by the thickness or number of layers [17]. The aforementioned thickness dependency was predicted computationally [18] and confirmed experimentally $[19,20]$. A possible explanation of this finding was attributed to the progressive improvement in cross-plane phonon transmission among the low frequency modes, as the number of layers increases [17]. Therefore, the interfacial thermal conductance between PDMS and graphene or borophene nanoflakes were calculated as a function of the number of layers, from one to six layers. To determine the interfacial thermal conductance values for PDMS/borophene and PDMS/graphene interfaces, the asymptotic value of convergence, occurring around six layers $[17,18]$ were considered. The constructed models, with the stacked layers of graphene and borophene ( 6 layers for both) over a block of PDMS polymer, is depicted in Figure 1. Equivalent models were used for lower numbers of layers. These systems include periodic boundary conditions in all directions, meaning that two interfaces between nanoflakes and polymer engage in the thermal transport. Moreover, periodic boundary conditions create a virtually continuous surface, without boundary issues, so the nanoflakes are designed to preserve the crystal periodicity even at boundaries. The specifications for a single-layer graphene model are $a=99.64 ; b=47.48 \AA$. This is equal to a contact area of about $4731 \AA^{2}$, which means a contact area of twice of the aforementioned value form with PDMS polymer in the thermal conductance calculations. The height of the systems varied from 84.6 to $105 \AA$, depending on layers stacking. Each layer of graphene, $3.4 \AA$ thick, was made of 1760 carbon atoms, bringing the total amount of atoms, including the PDMS to 32180 individual atoms. Similarly, for the borophene models, the contact area is about $4289 \AA^{2}$. In this case, 1260 atoms are included in each layer of borophene, bringing the total amount of atoms in the final models from 31680 for the single layer borophene to 39780 atoms for the case of six-layer borophene. The height varied with borophene layer thickness, therefore the size of composite systems along the stacking directions vary from $92 \AA$ for the monolayer to $112 \AA$ for the six-layers of borophene. The differences in cell topology between graphene and borophene models is due to the differences in the two-dimensional lattice between graphene and borophene. 
(a)

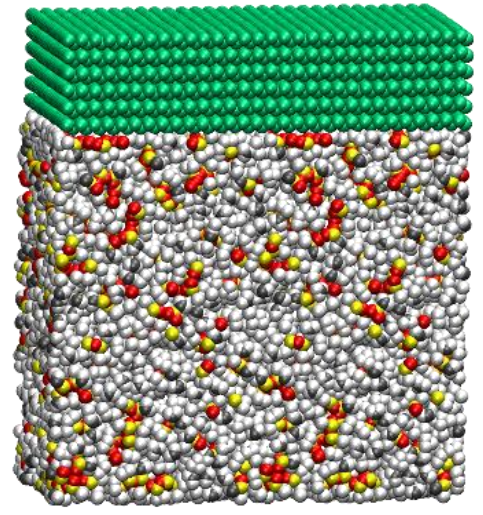

(b)

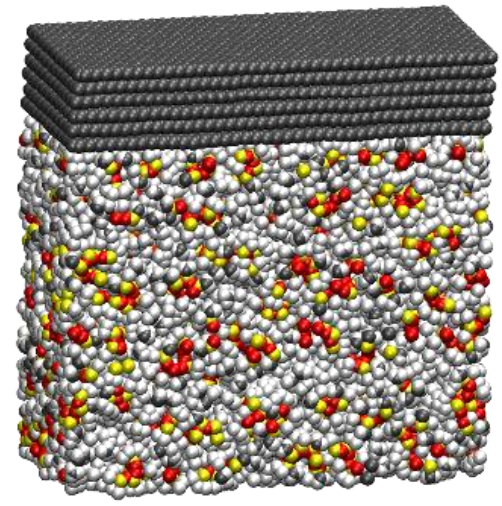

Figure 1. Constructed molecular models of six layers of borophene (a) and graphene (b) stacked over PDMS polymer. Color coding, includes; boron in green, carbon in dark grey, hydrogen in white, oxygen in red and silicon in yellow.

To calculate the thermal conductance between PDMS polymer and the layers of graphene and borophene platelets, the thermal equilibration method [18, 21-23] was adopted. At the beginning of the simulation, atomic velocities were initialized using the Maxwell Boltzmann distribution. During the equilibration process, the PDMS polymer and the stacked nanoplatelets temperature was kept at 300K and $350 \mathrm{~K}$, respectively, using the NVT method for $25 \mathrm{ps}$. In the second stage, we simulated the heat transfer; using a transient 500 ps step, where NVT was switched off and the polymer and nanofillers were allowed to reach the thermal equilibrium in microcanonical ensemble. Within this transient stage, the temperature variation of each phase is collected. The exponential fitting of the temperature difference allowed to determine the decay time $(\tau)$ of it. By knowing the masses of the polymer $\left(M_{p}\right)$ and filler $\left(M_{f}\right)$, the heat capacity of polymer $\left(C p_{p}\right)$, filler $\left(C p_{f}\right)$ and the interfacial area $(A)$, the interfacial thermal conductance across the interface $(\lambda)$, was calculated, using the following relation.

$$
\Delta T(t)=\Delta T(0) e^{\left[-\left(\frac{1}{M_{p} C p_{p}}+\frac{1}{M_{f} C p_{f}}\right)\right] \lambda A}
$$

The heat capacities values adopted in this work are $1.46 \mathrm{~J} \mathrm{~g}^{-1} \mathrm{~K}^{-1}$ for PDMS [24], $0.71 \mathrm{~J} \mathrm{~g}^{-1} \mathrm{~K}^{-1}$ for graphene [25] and $1.02 \mathrm{~J} \mathrm{~g}^{-1} \mathrm{~K}^{-1}$ for borophene [4]. The interfacial thermal conductance was calculated for the all systems. For every system, the calculations were conducted for 12 uncorrelated simulations and the temperatures were averaged. An example of thermal relaxation between borophene or graphene and PDMS are shown in Error! Reference source not found.. It is noticeable that during the relaxation the temperature difference between the nanofillers and the polymers, $\Delta T(t)$, decays exponentially. By conducting a fitting to the temperature difference, the interfacial thermal conductance was evaluated using the Eq. 1. 


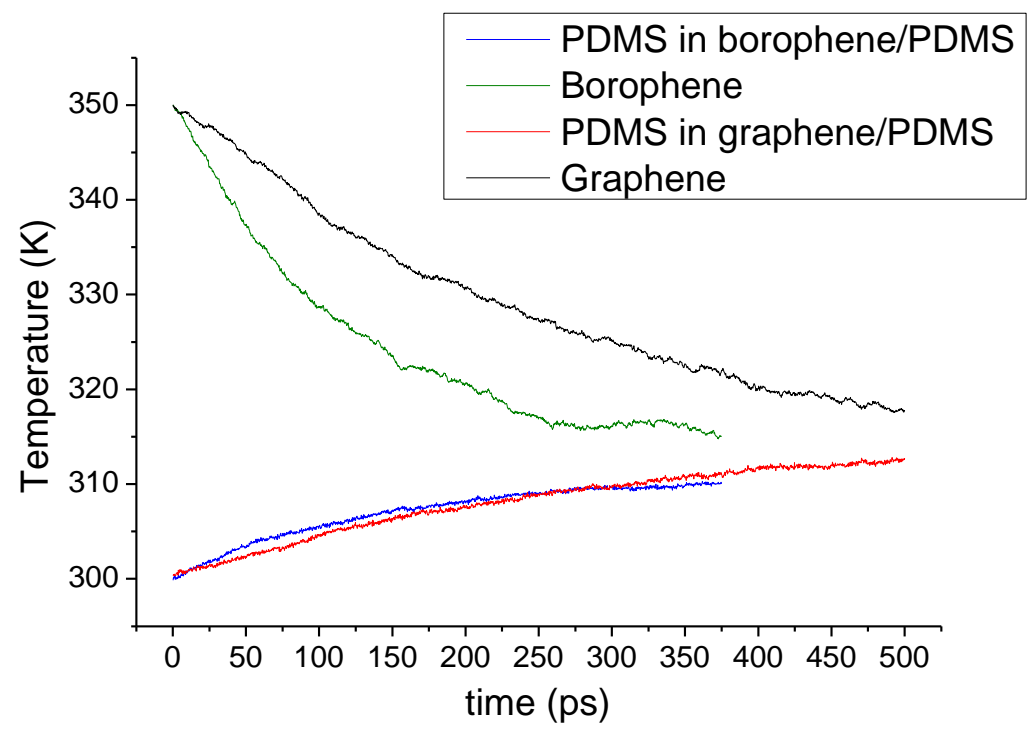

Figure 2: Averaged temperatures recorded during the heat transfer simulations for the six-layer nanosheets of graphene and borophene over the PDMS polymer.

In Figure 3, the predicted interfacial thermal conductance between graphene and borophene nanosheets and PDMS as a function of the number of layers are shown. Acquires results confirm that the interfacial thermal conductance increases from the single-layer to multilayered structures. For the case of graphene, it reaches a plateau and converges to a value of around $30 \mathrm{MW} \mathrm{m}^{-2} \mathrm{~K}^{-1}$ for the six and seven layered graphene/PDMS systems, in agreement with literature $[17,18]$. For the case of borophene, the interfacial thermal conductance sharply increases from the single-layer to threelayered structure, and for higher number of layers stay convincingly constant. Our results interestingly reveal that generally the borophene exhibits higher interfacial thermal conductance with the PDMS than graphene. For the case of borophene/PDMS, the interfacial thermal conductance converges to around $33 \mathrm{MW} \mathrm{m}^{-2} \mathrm{~K}^{-1}$, which is $10 \%$ more than that of the graphene/PDMS interface. This shows that the buckled structure of borophene and the high vibrations of boron atoms could enhance the heat transfer at the interface with the polymer as compared with the graphene.

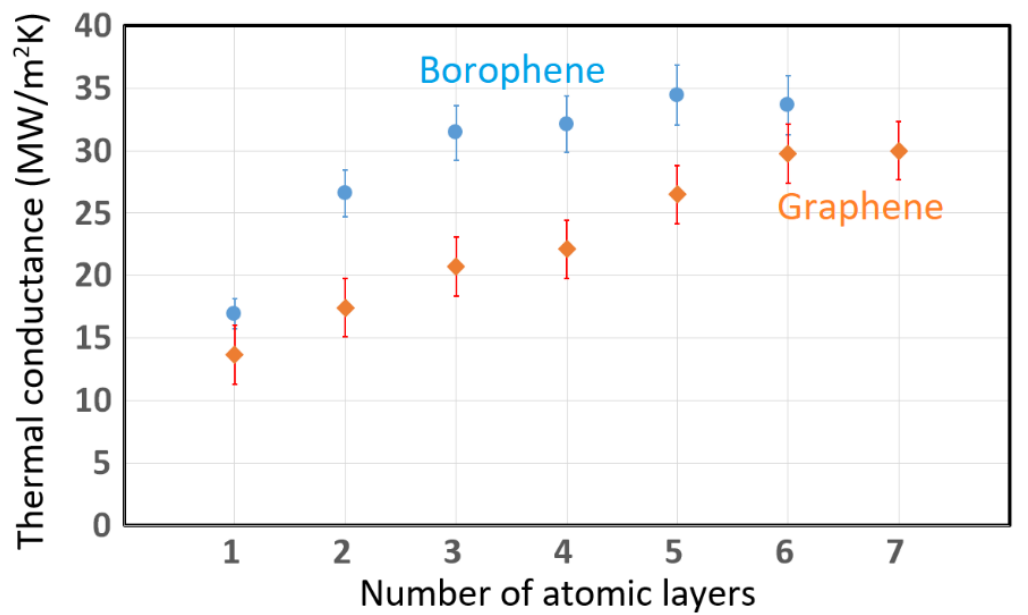

Figure 3. Interfacial thermal conductance between nanosheets and PDMS as a function of the number of layers of graphene and borophene. 
With calculated thermal conductance values, we are able to explore the effective thermal conductivity of macroscopic samples using the finite element approach. In this work, the thermal conductivity of multilayer graphene and borophene are assumed to be $2000 \mathrm{~W} \mathrm{~m}^{-1} \mathrm{~K}^{-1}[26,27]$ and $75 \mathrm{~W} \mathrm{~m}^{-1} \mathrm{~K}^{-1}[3]$, respectively. In these models, the converged value for the interfacial thermal conductance values are used to define the thermal contact properties between the fillers and polymer. Moreover, we assumed the disc geometry for the graphene and borophene, in which the aspect ratio is defined by the diameter to thickness ratio. The constructed models are all periodic, meaning that if a particle path a boundary surface of the RVE it enters from the opposite surface, and like that by putting the RVEs side by side all the fillers will show the perfect disc geometry. Fillers were randomly oriented and distributed without allowing their contact. Three filler loadings (volume fractions) of $1 \%, 2 \%$ and $4 \%$ were considered and the thickness of discs were assumed to vary from 1 to 10 and $100 \mathrm{~nm}$. In our simulations, for the evaluation of the effective thermal conductivity, a steady-state heat flux was imposed to the opposite surfaces. Within this layout, the heat flux passes through the meshed RVE, and form a temperature gradient inside the volume, as illustrated in Figure 4 . The effective thermal conductivity of PDMS based nanocomposites was calculated on the basis of one-dimensional Fourier's law, $k_{e f f}=L x q / \Delta T$, where $q$ is the applied heat flux, $L$ is the size of RVE and $\Delta T$ is the established temperature difference between the two ends of the model.

(a)

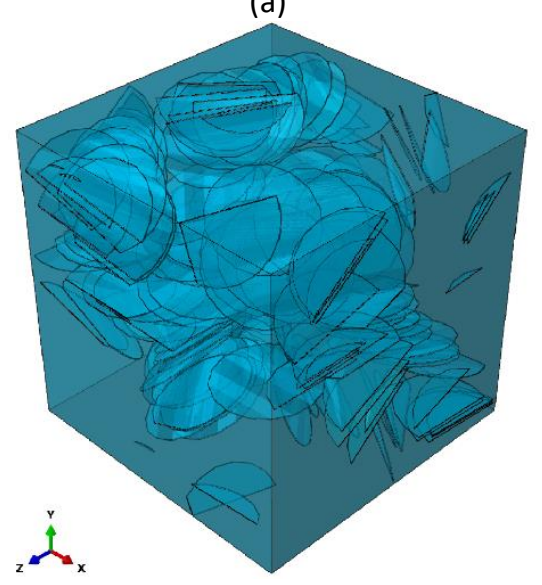

(b)

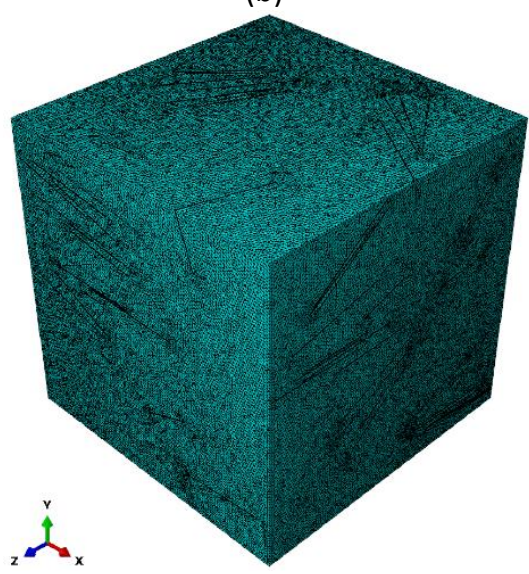

(c)

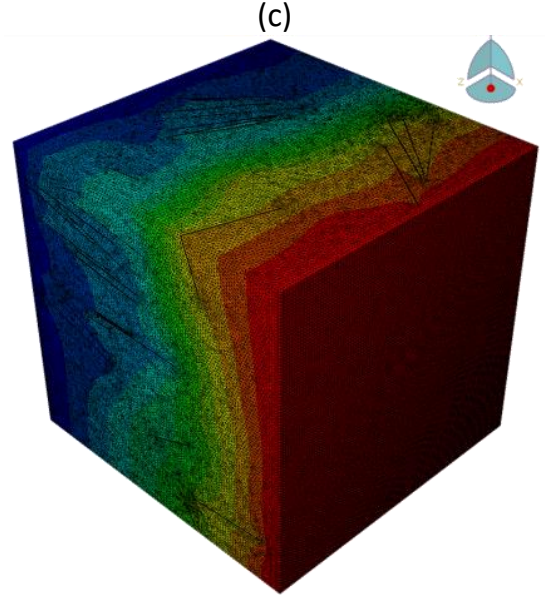

Figure 4. (a) An example of composite RVE including 150 flat disks with the aspect ratio 100 and $4 \%$ of volume concentration. (b) The 4-nodes linear tetrahedron shape mesh for the RVE (c) The established steady-state temperature profile created by applying the heat flux passing through the RVE (the color coding from red to blue depict hot to colder regions, respectively.

We first studied the effects of volume fraction and aspect ratio on the effective thermal conductivity of nanocomposites and the obtained results are shown in Figure 5. In these results, the thickness of nanosheets was assumed to be $1 \mathrm{~nm}$ (Figure 5a) and $100 \mathrm{~nm}$ (Figure 5b), taken as lower and upper bound values for experimentally exploitable nanoplates. All the predicted values for nanocomposites are clearly higher than the thermal conductivity of pure PDMS $\left(0.15 \mathrm{~W} \mathrm{~m}^{-1} \mathrm{~K}^{-1}\right)$, demonstrating that enhancement due to the presence of fillers with higher thermal conductivity. The volume fraction of the filler, as known from literature, confirmed as the typical parameter to tune to improve heat transfer in composite materials, where $4 \%$ of filler about doubles the TC compared to $1 \%$ condition. As expected 
by increasing the volume fraction and aspect ratio, the effective thermal conductivity of PDMS nanocomposites increases monotonically. By increasing the aspect ratio, the fillers allow more direct heat transfer along the composite. As known from literature [28], short particles featured by a low aspect ratio (25 and 50) confirmed to provide a smaller contribution than longer ones to improve thermal transport in composites. When particles with low aspect ratio are employed, the relatively thick layer of polymer interposed within the particles does now allow particles to create a three-dimensional path able to transport efficiently heat. As a very interesting finding, it is clear that borophene nanosheets, despite almost two orders of magnitude lower thermal conductivity than graphene, yield very close enhancement in the effective thermal conductivity in comparison with graphene, particularly for low content and small aspect ratio and thickness. For the nanocomposites with low concentration of fillers with small aspect ratios and thicknesses, borophene and graphene fillers show similar enhancement ratios. Nonetheless, graphene-based nanocomposites always show higher thermal conductivities than borophene counterparts. This reveals that the slightly different thermal conductance between borophene and PDMS could never compensate for their lower thermal conductivity compared to graphene. Results shown in Figure 5 clearly highlight that the difference due to the type of nanofillers on the enhancement of thermal conductivity become more pronounced for higher volume fractions, thickness and aspect rations for the nanofillers. By comparing the effects of thickness on the effective thermal conductivity, our results shown substantial effects. By increasing the thickness for a volume content of fillers, the effect of interfacial resistance decreases, resulting in a higher thermal conductivity. This finding suggests that, for polymer-based composites, significant improvement in thermal conductivity can be reached by improving the bonding between the fillers and polymer or, in other words, enhancing the thermal conductance at the interface. It is noticeable that borophene nanofillers with $100 \mathrm{~nm}$ thickness can yield distinctly higher enhancement in the effective thermal conductivity of nanocomposites than those made of graphene nanosheets with thickness of 1 $\mathrm{nm}$. Our results reveal that nanofillers with higher thicknesses and aspect ratio can result in higher thermal conductivities. It is also clear that nanofillers with higher thermal conductivities become more effective when their aspect ratio and thickness are larger. 


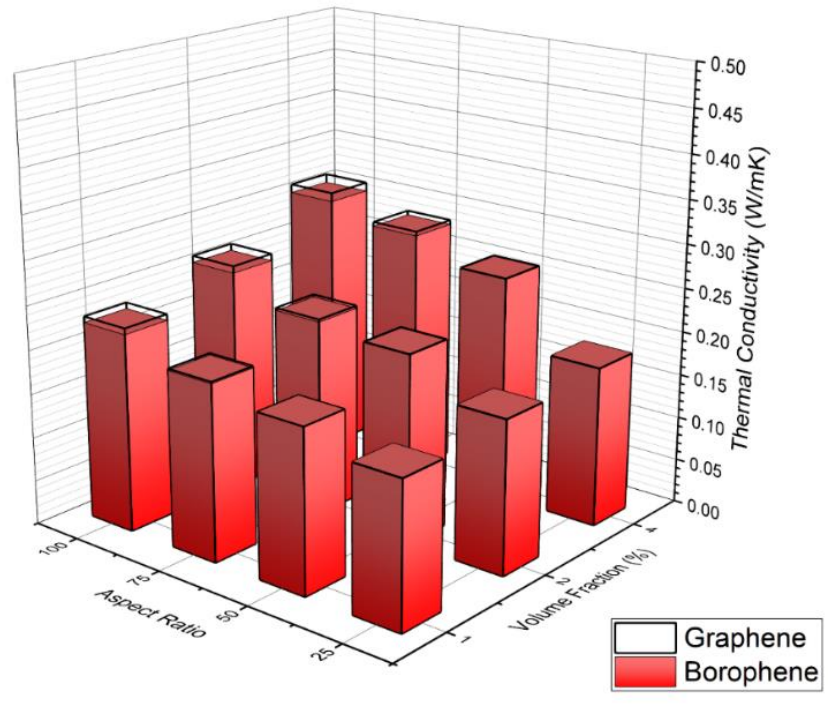

(a)

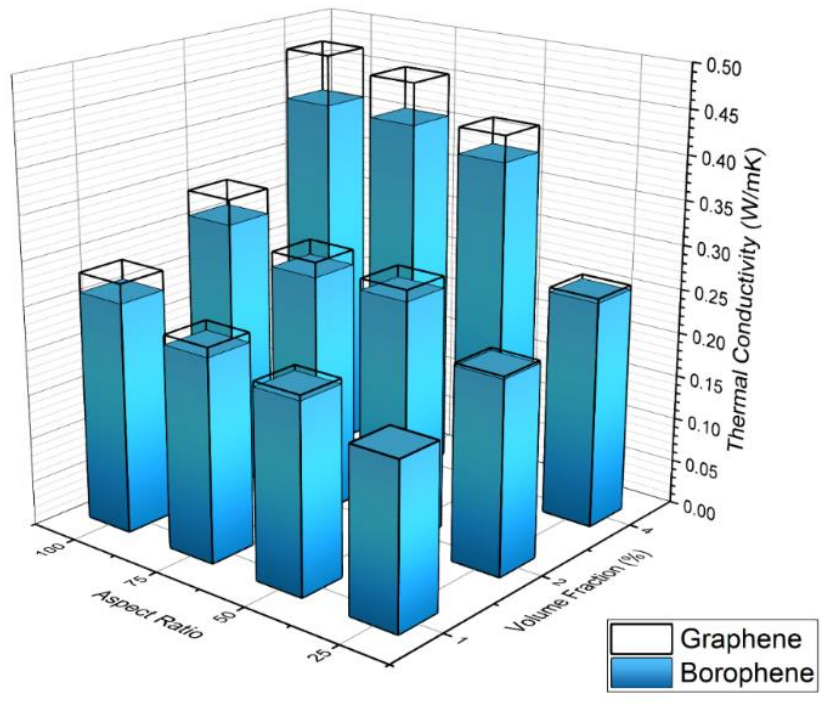

(b)

Figure 5. Effective thermal conductivity of PDMS nanocomposites as a function of nanofillers volume fraction and aspect ratio. The thickness of nanosheets was assumed to be (a) $1 \mathrm{~nm}$ and (b) $100 \mathrm{~nm}$.

\section{Concluding remarks}

In this study, a combined atomistic continuum multiscale modeling approach was developed to explore the effective thermal conductivity of polymers nanocomposites made of PDMS polymer and graphene and borophene nanofillers. This approach includes classical molecular dynamics simulations to investigate the interfacial thermal conductance between graphene/polymer and borophene/polymer interfaces. In the next step, the estimated interfacial thermal conductances were used in the finite element method simulations to evaluate the effective thermal conductivity of polymer nanocomposites at macroscopic level. We particularly examine the effects of nanofillers type, borophene or graphene and their volume content, geometry aspect ratio and thickness. Based on the Molecular Dynamics calculations the interfacial thermal conductance between the PDMS polymer and graphene and borophene were predicted to be $30 \mathrm{MW} \mathrm{m}^{-2} \mathrm{~K}^{-1}$ and $33 \mathrm{MW} \mathrm{m}^{-2} \mathrm{~K}^{-1}$, respectively. The acquired results confirm that the interfacial thermal conductance between nanosheets and polymer increases from the single-layer to multilayered nanofillers and finally converges. These estimated converged values were then employed to define contact thermal conductance in the finite element modeling of nanocomposites representative volume elements. As a very interesting finding, we show that borophene nanosheets despite almost two orders of magnitude lower thermal conductivity than graphene yield very close enhancement in the effective thermal conductivity in comparison with graphene, particularly for low content and small aspect ratio and thickness. This finding suggests that for polymer-based composites, significant improvement in the thermal conductivity can be reached by improving the bonding between the fillers and polymer, or in other words, by enhancing the thermal conductance at the interface. Additionally, the possibility to manufacture an effective percolation network by the exploitations of flake-to-flake junctions represents the smartest route to improve the thermal conductivity of nanocomposite. Taking into account the high electrical conductivity of 
borophene, our results therefore suggest borophene nanosheets as promising candidates for the improvement of polymers thermal and electrical conductivity.

\section{Acknowledgements}

This work has received funding from the European Research Council (ERC) under the European Union's Horizon 2020 research and innovation programme grant agreement 639495 - INTHERM - ERC-2014STG. Authors are greatly thankful to the VEGAS cluster at Bauhaus University of Weimar for providing the computational resources. B.M. appreciates the funding by the Deutsche Forschungsgemeinschaft (DFG, German Research Foundation) under Germany's Excellence Strategy within the Cluster of Excellence PhoenixD (EXC 2122, Project ID 390833453).

\section{References}

[1] K.S. Novoselov, A.K. Geim, S.V. Morozov, D. Jiang, Y. Zhang, S.V. Dubonos, I.V. Grigorieva, A.A. Firsov, Electric field effect in atomically thin carbon films, Science 306(5696) (2004) 666-9.

[2] A.K. Geim, Graphene: status and prospects, Science 324(5934) (2009) 1530-4.

[3] B. Mortazavi, M.-Q. Le, T. Rabczuk, L.F.C. Pereira, Anomalous strain effect on the thermal conductivity of borophene: a reactive molecular dynamics study, Physica E: Low-dimensional Systems and Nanostructures 93 (2017) 202-207.

[4] B. Peng, H. Zhang, H. Shao, Y. Xu, R. Zhang, H. Zhu, The electronic, optical, and thermodynamic properties of borophene from first-principles calculations, Journal of Materials Chemistry C 4(16) (2016) 3592-3598.

[5] S. Colonna, D. Battegazzore, M. Eleuteri, R. Arrigo, A. Fina, Properties of Graphene-Related Materials Controlling the Thermal Conductivity of Their Polymer Nanocomposites, Nanomaterials (Basel) 10(11) (2020).

[6] C.S. Boland, U. Khan, G. Ryan, S. Barwich, R. Charifou, A. Harvey, C. Backes, Z. Li, M.S. Ferreira, M.E. Mobius, R.J. Young, J.N. Coleman, Sensitive electromechanical sensors using viscoelastic graphene-polymer nanocomposites, Science 354(6317) (2016) 1257-1260.

[7] Y.-H. Zhao, Z.-K. Wu, S.-L. Bai, Study on thermal properties of graphene foam/graphene sheets filled polymer composites, Composites Part A: Applied Science and Manufacturing 72 (2015) 200-206.

[8] L. Tian, Y. Wang, Z. Li, H. Mei, Y. Shang, The thermal conductivity-dependant drag reduction mechanism of water droplets controlled by graphene/silicone rubber composites, Experimental Thermal and Fluid Science 85 (2017) 363-369. [9] Li, Zhang, Zhang, Thermal Conductivity of Graphene-Polymer Composites: Mechanisms, Properties, and Applications, Polymers 9(12) (2017) 437.

[10] F.E. Alam, W. Dai, M. Yang, S. Du, X. Li, J. Yu, N. Jiang, C.-T. Lin, In situ formation of a cellular graphene framework in thermoplastic composites leading to superior thermal conductivity, Journal of Materials Chemistry A 5(13) (2017) 61646169.

[11] K.M. Shahil, A.A. Balandin, Graphene-multilayer graphene nanocomposites as highly efficient thermal interface materials, Nano letters 12(2) (2012) 861-867.

[12] S. Plimpton, Fast parallel algorithms for short-range molecular dynamics, Journal of computational physics 117(1)

(1995) 1-19.

[13] LAMMPS website. <http://lammps.sandia.gov>).

[14] L. Lindsay, D.A. Broido, Optimized Tersoff and Brenner empirical potential parameters for lattice dynamics and phonon thermal transport in carbon nanotubes and graphene, Physical Review B 81(20) (2010).

[15] M.R. Weismiller, A.C. van Duin, J. Lee, R.A. Yetter, ReaxFF reactive force field development and applications for molecular dynamics simulations of ammonia borane dehydrogenation and combustion, J Phys Chem A 114(17) (2010) 5485-92.

[16] A.K. Rappe, C.J. Casewit, K.S. Colwell, W.A. Goddard, W.M. Skiff, UFF, a full periodic table force field for molecular mechanics and molecular dynamics simulations, Journal of the American Chemical Society 114(25) (1992) 10024-10035. [17] Z.-Y. Ong, Thickness-dependent Kapitza resistance in multilayered graphene and other two-dimensional crystals, Physical Review B 95(15) (2017) 155309. 
[18] B. Mortazavi, H. Yang, F. Mohebbi, G. Cuniberti, T. Rabczuk, Graphene or h-BN paraffin composite structures for the thermal management of Li-ion batteries: A multiscale investigation, Applied Energy 202 (2017) 323-334.

[19] F. Menges, H. Riel, A. Stemmer, C. Dimitrakopoulos, B. Gotsmann, Thermal transport into graphene through nanoscopic contacts, Phys Rev Lett 111(20) (2013) 205901.

[20] P. Yuan, C. Li, S. Xu, J. Liu, X. Wang, Interfacial thermal conductance between few to tens of layered-MoS2 and c-Si: Effect of MoS2 thickness, Acta Materialia 122 (2017) 152-165.

[21] S.T. Huxtable, D.G. Cahill, S. Shenogin, L. Xue, R. Ozisik, P. Barone, M. Usrey, M.S. Strano, G. Siddons, M. Shim, P. Keblinski, Interfacial heat flow in carbon nanotube suspensions, Nat Mater 2(11) (2003) 731-4.

[22] C.F. Carlborg, J. Shiomi, S. Maruyama, Thermal boundary resistance between single-walled carbon nanotubes and surrounding matrices, Physical Review B 78(20) (2008) 205406.

[23] Z.-Y. Ong, E. Pop, Molecular dynamics simulation of thermal boundary conductance between carbon nanotubes and SiO 2, Physical Review B 81(15) (2010) 155408.

[24] T.M. Madkour, J. Mark, Polymer data handbook, Oxford University Press New York, 1999.

[25] T. Tohei, A. Kuwabara, F. Oba, I. Tanaka, Debye temperature and stiffness of carbon and boron nitride polymorphs from first principles calculations, Physical Review B 73(6) (2006) 064304.

[26] H.Y. Chen, V.V. Ginzburg, J. Yang, Y.F. Yang, W. Liu, Y. Huang, L.B. Du, B. Chen, Thermal conductivity of polymer-based composites: Fundamentals and applications, Progress in Polymer Science 59 (2016) 41-85.

[27] Z.D. Han, A. Fina, Thermal conductivity of carbon nanotubes and their polymer nanocomposites: A review, Progress in Polymer Science 36(7) (2011) 914-944.

[28] N. Burger, A. Laachachi, M. Ferriol, M. Lutz, V. Toniazzo, D. Ruch, Review of thermal conductivity in composites:

Mechanisms, parameters and theory, Progress in Polymer Science 61 (2016) 1-28. 